\title{
El modelo pedagógico de la Carrera de Historia del Arte en la Universidad de Costa Rica (1970-2017): un estudio de caso
}

\author{
The Pedagogical Model of the Career \\ in Art History at the University of Costa Rica \\ (1970-2017): a Case Study
}

Ivannia Sofía Soto Monge

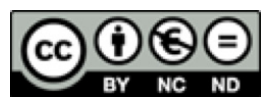

Esta obra está bajo una licencia Creative Commons Reconocimiento-No comercial-Sin Obra Derivada 
Dossier

\title{
El modelo pedagógico de la Carrera de Historia del Arte en la Universidad de Costa Rica (1970-2017): un estudio de caso' The Pedagogical Model of the Career in Art History at the University of Costa Rica (1970-2017): a Case Study
}

\author{
Ivannia Sofía Soto Monge ${ }^{2}$ \\ Universidad de Costa Rica \\ Costa Rica
}

Recibido: 10 de octubre de 2018 Aprobado: 10 de diciembre de 2018

\begin{abstract}
Resumen
La presente investigación muestra los resultados obtenidos del estudio y análisis del modelo pedagógico de la carrera de Historia del Arte en la Universidad de Costa Rica desde el año 1970 hasta el 2017. Su abordaje metodológico parte de la construcción de un estudio de caso, a través de la utilización de categorías y subcategorías de análisis, desarrolladas a partir del estudio teórico-conceptual sobre el modelo pedagógico, así como la información curricular encontrada en los planes de estudio de la carrera. A partir de los resultados obtenidos, se concluye que existe una ausencia de fundamentos teóricos propios de la educación que sustenten la existencia de un modelo pedagógico en la carrera.
\end{abstract}

Palabras clave: Historia del Arte; pedagogía; modelo pedagógico; análisis curricular; estudio de caso

1 El análisis que plantea este artículo proviene de los resultados obtenidos en el primer apartado del estudio realizado sobre el modelo pedagógico de la carrera de Historia del Arte, con la cual la autora dio por finalizado, en el 2018, su formación en Licenciatura en Historia del Arte por la Universidad de Costa Rica. Dicho documento se denomina La carrera de Historia del Arte en la Universidad de Costa Rica (1970-2017): un estudio de caso sobre su modelo pedagógico.

2 Profesora de Historia de la Fotografía en la Universidad Creativa de Costa Rica. Licenciada en Historia del Arte y Egresada de la Licenciatura en Ciencias de la Educación con Énfasis en Administración de la Educación no Formal, ambas en la Universidad de Costa Rica. Correo electrónico: ivannia.soto@ucr.ac.cr

ESCENA. Revista de las artes, 2019, Vol. 79, Núm. 1, pp. 249-274 ISSN 2215-4906 


\begin{abstract}
This research shows the results obtained from the study and analysis of the pedagogical model of the Art History career at the University of Costa Rica, from 1970 to 2017. Its methodological approach is based on the construction of a case study through the use of categories and subcategories of analysis, developed from the theoretical-conceptual study of the pedagogical model, as well as the curricular information found in the study plans of the career. Based on the results obtained, it is concluded that there is an absence of theoretical foundations specific to education that support the existence of a pedagogical model in the career.
\end{abstract}

Key words: Art History; pedagogy; pedagogical model; curricular analysis; case study 


\section{Introducción}

El estudio de la Historia del Arte se ha encontrado históricamente vinculado a la investigación de las manifestaciones artísticas visuales, desde la constitución misma del objeto, el artista y su relación sociocultural con el entorno. A partir de esta realidad, el acercamiento a un estudio o investigación que vincule a la Historia del Arte con la práctica educativa y docente es novedoso. Desde esta perspectiva, la problematización del diseño curricular tradicional, así como el cuestionamiento sobre cómo y qué enseñamos en la Historia del Arte, se propone como un campo de investigación con espacio para la innovación. El interés por plantear una investigación centrada en aspectos pedagógicos y curriculares propios de la carrera de Historia del Arte respondió a la convicción de que existe una estrecha relación entre el quehacer académico, la educación y la Historia del Arte como disciplina.

La investigación que se desarrolla en este artículo, se encuentra dividida en los siguientes apartados. Inicialmente, se proporciona una aproximación teórica al estudio de la teoría curricular y al modelo pedagógico, como elemento que constituye el programa formal educativo, a partir de tres interrogantes: ¿Qué enseñar? ¿Cómo enseñar? ¿Qué y cómo evaluar? El siguiente apartado corresponde a la metodología utilizada para llevar a cabo el estudio y análisis propuestos. Basado en la elaboración de un estudio de caso, se propuso la construcción de categorías y subcategorías de análisis que permitiesen una revisión clara y pormenorizada de los elementos que constituyen un modelo pedagógico desde las metas educativas, los contenidos, la estructura curricular, la metodología de enseñanza y la evaluación, todas ellas, comprendidas desde la revisión de los planes de estudio que han constituido la carrera de Historia del Arte. El tercer apartado presenta el análisis pormenorizado de cada una de las categorías y subcategorías desarrolladas en la metodología, seguido de los resultados finales o hallazgos encontrados sobre el modelo pedagógico de la carrera de Historia del Arte en la Universidad de Costa Rica. Por último, encontramos el apartado de las conclusiones, el cual se deriva de los hallazgos más significativos estudiados en el análisis de las categorías y los resultados finales.

\section{Modelo pedagógico en la educación}

La educación, en sus distintas estructuras, se encuentra integrada por una serie de parámetros pedagógicos que han sido construidos para establecer un control procedimental y estratégico de enseñanza, en los distintos espacios educativos. Para Ortiz (2013), un modelo pedagógico parte de una construcción epistémica y formal, producto de un fundamento social, político e ideológico que determina los procedimientos de enseñanza en un contexto y periodo determinado. Estos suelen ser entendidos, también, como procesos educativos que buscan la formación del individuo, a partir de una práctica pedagógica, un programa y un perfil institucionalizado.

ESCENA. Revista de las artes, 2019, Vol. 79, Núm. 1 (julio-diciembre), pp. 249-274 
Para las autoras Pinto y Castro (2008), un modelo pedagógico parte, directamente, de "las prácticas cotidianas que se realizan dentro del aula, las cuales son percibidas como manifestaciones materiales de parámetros pedagógicos que, de manera implícita, se encuentra en las acciones didácticas de los maestros" (p. 2). Esto quiere decir, además, que los docentes son partícipes de un discurso teórico implícito que da soporte a sus prácticas de enseñanza. Otros autores como Florez, citado por Pinto y Castro (2008), definen los modelos pedagógicos como:

Formas particulares de interrelación entre los parámetros pedagógicos. El sentido de parámetros pedagógicos es, en el concepto de este autor, el trasfondo de explicaciones acerca de una concepción del ser humano específica y de una idea claramente determinada de la sociedad. De igual manera, este enfatiza la necesidad de la aplicación de un análisis riguroso con métodos sistemáticos en el estudio de los modelos pedagógicos (p. 2).

Al tomar en cuenta lo expuesto anteriormente, podríamos argumentar que un modelo pedagógico está constituido por los siguientes aspectos: las metas educativas; los contenidos de enseñanza; el estilo de relación entre profesor-alumno; los métodos de enseñanza; los conceptos básicos de desarrollo social y el tipo de institución educativa. "No es más que un paradigma que sirve para analizar, interpretar, comprender, orientar, dirigir y transformar la educación" (Ortiz, 2013, p. 70). Dentro de la estructura de la educación superior, el modelo pedagógico se vuelve una construcción epistémica que responde, asiduamente, a los procesos relativos a las cuestiones pedagógicas. Pero, especialmente, a las tendencias teóricas y metodológicas de la institución. En las universidades se suelen definir los parámetros pedagógicos dentro de las unidades académicas o de enseñanza. Por esto, podríamos discutir que, en ellas, se integran los siguientes componentes: un currículo formativo, cómo enseñar y cómo aprender, las metodologías utilizadas para impartir los contenidos de los programas y sus aplicaciones didácticas. Junto a esto, la puesta en práctica de estas orientaciones teóricas, metodológicas y prácticas responden, también, a los elementos activos de los programas: el profesor como el sujeto encargado de llevar a cabo lo que la institución le asigna y el estudiante, núcleo de la enseñanza y de la producción de conocimiento. Según Porlán (1983), citado por Gómez y Polania (2008), la identificación de las características que determinan un modelo pedagógico son construidas a partir de tres cuestionamientos básicos, las cuales permiten establecer un proceso educativo eficaz e integral con las necesidades sociales, políticas, económicas y culturales del contexto. Exponemos estas tres interrogantes en la Tabla 1 que se muestra a continuación: 
Tabla 1. Tabla de las interrogantes que construyen el modelo pedagógico

\begin{tabular}{|c|c|l|}
\hline \multicolumn{2}{|c|}{ Modelo Pedagógico } \\
\hline ¿Qué enseñar? & Enfoque & $\begin{array}{l}\text { Nos habla de los contenidos, su relevancia } \\
\text { y el orden en el cuál los desarrollamos. }\end{array}$ \\
\hline ¿Cómo enseñar? & Metodología & $\begin{array}{l}\text { Apunta a los métodos, medios y recursos } \\
\text { que utiliza el docente en la dinámica de } \\
\text { enseñanza-aprendizaje. }\end{array}$ \\
\hline $\begin{array}{c}\text { ¿Qué y cómo } \\
\text { evaluar? }\end{array}$ & $\begin{array}{l}\text { Formas de } \\
\text { evaluación }\end{array}$ & $\begin{array}{l}\text { Se refiere al momento, instrumentos y contenidos } \\
\text { revisados, desde el inicio del proceso educativo. } \\
\text { Las estrategias didácticas y la relación } \\
\text { enseñanza-aprendizaje están activamente } \\
\text { presentes en esta instrucción. }\end{array}$ \\
\hline & \multicolumn{2}{|c}{} \\
\hline
\end{tabular}

Fuente. Elaboración propia a partir de la información extraída de Gómez y Polania (2008).

A partir de los diferentes criterios conceptuales que hemos mencionado, nos queda claro que, hablar sobre modelo pedagógico involucra tomar en cuenta las propuestas teóricas y prácticas orientadas a la elaboración y análisis de los programas de estudio. Así como la sistematización del proceso de enseñanza-aprendizaje y las dinámicas de producción de conocimiento generadas de la interacción entre el profesor y el alumno.

\section{Metodología}

La investigación realizada partió del desarrollo de un análisis cualitativo de carácter descriptivo y exploratorio, sobre el objeto de estudio: el modelo pedagógico de la carrera de Historia del Arte de la Universidad de Costa Rica (UCR) en cuarenta y siete años de existencia. Descriptivo, ya que se realizó un estudio y recopilación de información sobre el objeto antes definido y exploratorio, debido a que este nunca ha sido propiamente examinado o estudiado. La metodología que se eligió parte del estudio de caso, este es definido por Hernández, Fernández y Baptista (2010) como "estudios que, al utilizar los procesos de investigación cuantitativa, cualitativa o mixta analizan, profundamente, una unidad para responder al planteamiento del problema, probar hipótesis y desarrollar alguna teoría" ( $p$. 163). La flexibilidad a la hora de determinar la manera en la cual se divide la investigación, su capacidad de análisis crítico y profundo hacen al estudio de caso una metodología con ventajas importantes. 
Por otro lado, la triangulación de la información le permite al investigador identificar los patrones que concurren, para poder desarrollar o corroborar una interpretación general del objeto de la investigación. Para el caso de esta investigación, la triangulación se efectuó a partir de la revisión y contrastación de la documentación administrativa y educativa sobre los distintos planes de estudio (fuente primaria), las entrevistas realizadas y la teoría educativa estudiada sobre el modelo pedagógico. De esta forma, se logró un análisis a profundidad de las evidencias y los vacíos encontrados y referenciados. Aunado a lo anterior, se estructuró la información a partir de categorías y subcategorías de análisis, como técnica para analizar la información recolectada.

\section{Criterios para la selección de datos}

Los criterios utilizados para la selección de las fuentes de información se dividieron en dos secciones específicas. La primera de ellas fue la recolección de documentos administrativos, educativos y de tipo operacional, que definieran, a nivel institucional, los parámetros por los cuáles están normadas las distintas carreras de la UCR. En el caso de estudio de la documentación fue de tipo curricular y educativa de la carrera de Historia del Arte. Se solicitó la documentación archivada por la Vicerrectoría de Docencia, el Centro de Evaluación Académica y El Archivo Universitario de la UCR ${ }^{3}$. Una vez revisada la documentación, se constataron vacíos importantes en la información que estos archivos poseían. Se determinó, de esta forma, que la mejor manera de recabar la información ausente de la documentación era mediante el instrumento de la entrevista.

Asimismo, se decidió que los participantes de esta muestra debían cumplir con una serie de criterios específicos, los cuáles se muestran a continuación. El primer criterio de selección determinó que los y las participantes de la muestra debían ser personas graduadas del bachillerato y la licenciatura de la carrera de Historia del Arte de la UCR. Se planteó de esta manera, pues estos individuos tendrían la experiencia y conocimiento adquirido en la formación disciplinar de la carrera, así como la estructura curricular bajo la cual recibieron su formación. En el segundo criterio propuesto estableció la necesidad de que los y las participantes ejercieran o hubieran ejercido como docentes dentro de la carrera o en otro espacio de educación superior que plantease cursos afines a los impartidos dentro de la carrera de Historia del Arte. Se definió así, ya que son los docentes dentro de una carrera universitaria quienes conocen a profundidad la estructura curricular (planes de estudio) que compone su disciplina, los programas y contenidos de los cursos, así como la experiencia docente en el aula.

3 Se debe señalar que estos documentos fueron las únicas fuentes encontradas luego de realizar la consulta y solicitud a los departamentos universitarios correspondientes.

ESCENA. Revista de las artes, 2019, Vol. 79, Núm. 1 (julio-diciembre), pp. 249-274 
Una vez determinadas las particularidades de los y las partícipes de la muestra fue necesario establecer el tipo de entrevista y los contenidos de esta. Se decidió realizar una entrevista semiestucturada, a modo de guion, dividida en dos grandes temas, que, a su vez, se segmentaron en cuatro pequeños subtemas relacionados a la teoría educativa. A continuación, se presenta en la Tabla 2, los temas y subtemas que compusieron la entrevista:

\section{Tabla 2. Información consultada en la entrevista}

\begin{tabular}{|c|c|c|}
\hline Temas & $\begin{array}{l}\text { 1. Experiencia personal dentro de la } \\
\text { carrera de historia del arte en la UCR }\end{array}$ & $\begin{array}{l}\text { 2. Modelo pedagógico dentro de la } \\
\text { carrera de historia del arte de la UCR }\end{array}$ \\
\hline \multirow{4}{*}{ Subtemas } & $\begin{array}{l}\text { 1.1. Período en el cual desarrolló sus } \\
\text { estudios dentro de la carrera de } \\
\text { Historia del Arte. }\end{array}$ & $\begin{array}{l}\text { 2.1. Instrumentos y modalidades } \\
\text { de enseñanza. }\end{array}$ \\
\hline & 1.2. Expectativas sobre la carrera. & $\begin{array}{l}\text { 2.2. Desde su experiencia dentro de la } \\
\text { carrera de Historia del Arte, ¿considera } \\
\text { usted que la carrera posee un modelo } \\
\text { pedagógico determinado? }\end{array}$ \\
\hline & $\begin{array}{l}\text { 1.3. El programa de estudios y su } \\
\text { finalidad formativa. }\end{array}$ & $\begin{array}{l}\text { 2.3. Si existe un modelo, cuáles serían } \\
\text { las ventajas y/o desventajas de este } \\
\text { modelo de enseñanza. }\end{array}$ \\
\hline & $\begin{array}{l}\text { 1.4. Alcances y limitaciones dentro de } \\
\text { la formación disciplinar }\end{array}$ & $\begin{array}{l}\text { 2.4. Formas de enseñanza utilizadas } \\
\text { dentro de la disciplina de la Historia del } \\
\text { Arte en la UCR (si las hay o no). }\end{array}$ \\
\hline
\end{tabular}

Fuente. Elaboración propia.

\section{Categorías de análisis como fuentes teórico-metodológicas}

Para una comprensión más clara del objeto de estudio, se propuso realizar un análisis de la información recabada de las fuentes documentales y las entrevistas realizadas, a partir de la configuración de una serie de categorías y subcategorías de análisis. Se partió de la sistematización de los criterios que permiten la elaboración de un modelo pedagógico, para así, construir activamente, los elementos indispensables dentro del acto pedagógico: características formativas, curriculares, metodológicas y evaluativas. Bajo estos criterios, el análisis de modelo pedagógico tuvo como objetivo identificar lo que Ortiz (2013) determina 
como las aspiraciones dentro del currículo educativo. De esta forma, se propusieron las siguientes categorías observadas en la Tabla 3:

Tabla 3. Categorías y subcategorías de análisis

\begin{tabular}{|c|c|c|}
\hline & \multicolumn{2}{|c|}{ Categorías de análisis } \\
\hline Interrogante activa & Categoría & Subcategorías \\
\hline \multirow{2}{*}{ ¿Qué debo enseñar? } & Enfoque pedagógico & Contenidos de la enseñanza. \\
\cline { 3 - 3 } ¿A quién enseño? & Currículo formativo. \\
\hline ¿Cómo enseño? & Metodología pedagógica & Metodología o estrategia de enseñanza. \\
\cline { 3 - 3 } & ¿Qué evaluar? & Instrumentos de enseñanza. \\
\hline ¿Cómo evaluar? & Evaluación de la enseñanza & Instrumentos de evaluación. \\
\hline
\end{tabular}

Fuente. Elaboración propia.

La primera categoría de análisis definida como "enfoque pedagógico" surge de los cuestionamientos teóricos expuestos acerca del modelo pedagógico, observados en los textos de Gómez y Polania (2008) y Pinto y Castro (2008). En estos se establece la delimitación formal del contenido, a partir de las interrogantes ¿qué debo enseñar? y ¿a quién enseño?, las cuales determinan el accionar del enfoque teórico y metodológico en la enseñanza. A partir de esto, configuramos tres subcategorías que se desprenden del enfoque pedagógico. Estas son: a) las metas educativas de la enseñanza, logradas mediante el aprendizaje de los contenidos; b) los contenidos de la enseñanza son los insumos teóricos, metodológicos y prácticos y c) el currículo formativo que representa la secuencia lógica de contenidos a nivel de curso o programa de estudios.

La segunda categoría titulada "metodología pedagógica" responde a la ejecución en clase de los contenidos propios del programa o plan de estudios, así como a la secuencia procedimental que el profesor establece, para desarrollar los contenidos dentro de los cursos. Conceptualizamos esta categoría bajo la propuesta de Gómez y Polania (2008), en la cual se comprende como "expresiones individuales de cada profesor... el conjunto de rasgos propios y la forma particular de actuar de un docente en su cotidiano del aula" ( $p$. 50). Dentro de ella, se busca dar respuesta a la pregunta ¿cómo enseño? partiendo de la 
delimitación de las siguientes subcategorías: 1) metodología o estrategia de enseñanza, con la cual el profesor imparte los contenidos educativos y 2) los instrumentos de enseñanza utilizados para llevar a cabo la implementación teórica o práctica de esas metodologías.

La tercera y última categoría de análisis propuesta como "evaluación de la enseñanza" se desprende, directamente, de las interrogantes que todo proceso evaluativo en la enseñanza debe responder, nos referimos a ¿qué evaluar? y ¿cómo evaluar? La subcategoría que la compone son los instrumentos de evaluación utilizados para determinar cómo y bajo qué condiciones se construyen los procesos evaluativos (Mora, 2004).

\section{Categoría 1. Enfoque pedagógico}

\section{Subcategoría 1.1 Metas Educativas}

El primer plan de estudios de 1970 propone una formación para bachillerato basada en las artes, en la historia universal, los idiomas, la historia del arte y, por último, la antropología (Consejo Universitario, 1970). Se observa, en este caso, una vinculación de los referentes educativos con las artes plásticas, en general, ya que la mayoría de los cursos se encontraban dirigidos a una formación en este ámbito. Por otro lado, los cursos dedicados a la historia del arte abarcaban temas de la tradición del arte europeo. Estos cursos partían de una estructura espacial y cronológica bien definida, al dar inicio en el arte antiguo, hasta llegar al siglo XX y las vanguardias artísticas. Esta disposición cronológica se ha mantenido como parte de la estructura base de los demás planes de estudio. Además, se ha complementado con los cursos de Filosofía e Historia Universal.

A pesar de esto, el plan contemplaba, únicamente, cuatro cursos dedicados a la Historia del Arte. Mientras que, el resto de la estructura curricular veló, principalmente, por la implementación de una enseñanza donde primaba la visión de las artes plásticas occidentales de la pintura, escultura y grabado. Al referirse al primer plan de estudios de 1970, uno de los sujetos entrevistados discute sobre la simpleza de este en un inicio. No había posibilidad de profundizar en temas más allá de los artistas y movimientos artísticos que se enseñaban en estos cursos, lo que provocó un acceso limitado al estudio de otras áreas del conocimiento más especializadas (Comunicación personal, 20 de abril del 2017). No existe aún el interés por una profesionalización de la disciplina. La finalidad formativa o metas educativas de este primer plan de estudios para el bachillerato partían, directamente, de la formación artística en un primer plano, lo cual dejó un mínimo de tiempo para el conocimiento sobre la Historia del Arte. Más allá del fomento de la capacidad teórica y metodológica en el estudio de la Historia del Arte, se incentiva la praxis de las técnicas artísticas para la producción gráfica. Se puede afirmar que este primer plan de estudios buscó formar artistas con conocimientos generales sobre Historia del Arte, y no Historiadores del Arte. 
Para el plan de 1985, se observa una reducción significativa de las asignaturas dedicadas a la formación artística, así como un aumento en los cursos de Historia del Arte (separados ya en contenidos más claros). Los cursos de Historia Universal se mantienen relativamente estables. Tenemos, ahora, una construcción curricular que apela de forma contundente al aprendizaje de una Historia del Arte basada en un paradigma positivista de la escuela alemana del siglo XVIII, donde prima una visión eurocentrista formal de los estilos artísticos (Efland, 2002).

Para el plan de estudios del 2007 y el cambio parcial del 2013 se mantienen las mismas características ligadas a una formación en el ámbito de la Historia del Arte Occidental. Solamente que, ahora, se complementa con cursos de Filosofía e Historia Universal. Las metas educativas parecen variar en cuanto a la inserción de una formación entre la Historia del Arte, la Historia y la Filosofía.

Por último, el plan de estudios aprobado para el 2017 parece haber intentado ordenar los intereses educativos para la formación del profesional en Historia del Arte. Al observar el documento de esta malla curricular constatamos que mantiene, todavía, el mismo ordenamiento de contenidos referidos a la Historia del Arte europea, solo que ahora los cursos deben llevarse en una secuencia lógica entre Historia del Arte, Historia y Filosofía (Centro de Evaluación Académica, 2017). Cabe mencionarse que, el plan aún es muy reciente para ser analizado en su efectividad y en la medición del cumplimiento de las metas educativas que se propone.

\section{Subcategoría 1.2 Contenidos de la enseñanza}

Los contenidos de la enseñanza fueron más difíciles de identificar, debido a que no aparecen de forma explícita en la documentación recabada. Es por esto que se recurrió a las entrevistas realizadas. La intención principal era ubicar los programas de los cursos para cada una de las mallas curriculares (1970, 1985, 2007, 2013 y 2017) en la Escuela de Artes Plásticas, pero, la unidad académica no poseía esta información. Esta situación dificultó el análisis de esta sección. Se observó que la mayoría de los contenidos de los cursos de Historia del Arte, en todos los planes de estudios analizados, se encontraban enmarcados dentro de los límites historiográficos de las artes plásticas occidentales. Es decir, el enfoque de los contenidos educativos parte del paradigma del positivismo mencionado en las metas educativas. Este se centra, principalmente, en un orden secuencial del arte europeo en sus distintas fases. Un orden secuencial que se repite en los cursos referentes al continente americano y demás temas que cubren los cursos optativos (esto se observa más adelante en el "currículo formativo"). 
En la tabla 4 se puede observar la distribución de cursos por contenidos de las materias sobre Historia del Arte, impartidos durante la carrera, y sus modificaciones según el periodo. Esto nos brinda un panorama más concreto sobre cómo se comportaron los cambios curriculares, cómo fueron afectados los contenidos de los cursos y cómo se perciben esas reformas frente a la nueva malla curricular del 2017.

Tabla 4. Cantidad de cursos por contenido o tema de estudio de los distintos planes de la Carrera de Historia del Arte $^{4}$

\begin{tabular}{|l|l|l|l|l|l|}
\hline \multicolumn{7}{|c|}{ Planes de Estudio de la carrera de Historia del Arte } \\
\hline \multicolumn{1}{|c|}{ Contenidos de cursos } & $\begin{array}{c}\text { Plan de } \\
1970\end{array}$ & $\begin{array}{c}\text { Plan de } \\
1985\end{array}$ & $\begin{array}{c}\text { Plan del } \\
2007\end{array}$ & $\begin{array}{c}\text { Plan del } \\
2013\end{array}$ & $\begin{array}{c}\text { Plan del } \\
2017\end{array}$ \\
\hline $\begin{array}{l}\text { Arte occidental (Europa y } \\
\text { Estados Unidos) }\end{array}$ & 4 & 8 & 9 & 11 & 9 \\
\hline $\begin{array}{l}\text { Arte costarricense, americano } \\
\text { y latinoamericano }\end{array}$ & 1 & 2 & 3 & 4 & 6 \\
\hline Cursos teóricos & 0 & 1 & 5 & 7 & 7 \\
\hline Cursos de metodología e investigación & 0 & 0 & 0 & 0 & 0 \\
\hline Lecturas dirigidas & 0 & 3 & 4 & 4 & 4 \\
\hline Optativos de Historia del Arte & 0 & 5 & 0 & 1 & 4 \\
\hline Otros & 0 & 0 & 3 & 3 & 4 \\
\hline
\end{tabular}

Fuente. Elaboración propia a partir de la información tomada del Consejo Universitario (1970), la Vicerrectoría de Docencia (2007) y el Centro de Evaluación Académica (1985; 2013; 2017).

Con la implementación de nuevas estructuras curriculares, a partir del plan de 1985, se observa como hay un aumento significativo de cursos delimitados por contenidos del arte occidental europeo y de Estados Unidos. Esto reafirma las intenciones educativas señaladas en párrafos anteriores, sobre el interés por formar al estudiante de Historia del Arte bajo una base del academicismo occidental. Las modificaciones durante el periodo del plan de

${ }^{4}$ Los cursos teóricos representan cursos donde los contenidos buscan acercar al estudiante a fundamentos de carácter epistémico, postulados filosóficos y teóricos para el análisis de contenidos dentro de la Historia del Arte. Estos cursos son: Teoría del Arte I, Teoría del Arte II, Semiótica del Arte, Lectura y Análisis de la Imagen, Iconografía I y II (ahora Iconografía), Crítica de Arte, y Los Estudios Visuales: Visualidad y Producción Cultural. Además, el tema "Otros" representa los cursos que no tienen una sola área temática o espacio artístico como: Museología, Mitología en el Arte, Historia de la Fotografía, Mujeres Artífices y Artista, y Arte y Sociedad. 
estudios del 2007 y luego en el 2013 buscaron implementar una enseñanza que compartiese contenidos, simultáneamente ordenados, entre cursos de Historia del Arte, Historia Universal y cursos de Filosofía (Escuela de Filosofía). Estos criterios se cumplieron hasta la entrada en vigencia del actual plan de estudios del 2017 (Centro de Evaluación Académica, 2017).

Uno de los aspectos que más sobresalen en la tabla 4 es la ausencia total de contenidos sobre metodologías de investigación, en cada uno de los planes de estudio. Parece ser que no ha existido un interés por solventar las falencias que provoca esta situación en el estudiante de la carrera de Historia del Arte en la UCR, a nivel de producción académica y de formación de competencias. Esto se refleja, también, en la calidad de las producciones investigativas relacionadas con el trabajo final de graduación (Entrevista Sujeto 4, comunicación personal, 19 de mayo del 2017). Es importante señalar que un historiador del arte que carece de habilidades para la investigación, pierde el sentido de su profesión. Es claro que se ha logrado un cambio significativo en cuanto al ordenamiento y estructura del currículo del bachillerato y licenciatura de la carrera. Pero, aún interesa el tema de cómo responden los procesos pedagógicos a las necesidades de un actual historiador del arte en Costa Rica.

\section{Subcategoría 1.3 Currículo Formativo}

El primer plan de estudios creado para la carrera de Historia del Arte surge con la aprobación del acta del Consejo Universitario del 28 de setiembre de 1970 (Consejo Universitario, 1970). En esta, se establece la primera división y estructura formal de cursos que integran la carrera en la modalidad de bachillerato. Es importante señalar que, este documento presenta, únicamente, la modalidad de bachillerato con su plan de estudios correspondiente. Sin embargo, gracias al aporte del Sujeto 2 entrevistado, se pudo constatar que sí existió un plan de licenciatura que, al parecer, se constituyó a lo largo de la década de los setentas (Entrevista Sujeto 2, comunicación personal, 20 de abril del 2017).

Los cursos que integran este plan de estudios, presentados en la tabla 5, se encuentran distribuidos en siete áreas temáticas, en las cuales sobresalen los cursos de carácter práctico de artes, lenguas, historia y sus cursos complementarios ${ }^{5} \mathrm{y}$, por último, historia del arte. Las áreas de antropología y letras representan un reducido margen de cursos de la malla curricular del periodo. En la tabla 5 se puede observar un ordenamiento de cursos de forma secuencial. Sobresalen, por un margen más amplio, los de Artes, que son aquellos de carácter práctico impartidos para la formación de profesionales dentro de las artes plásticas.

${ }^{5}$ Los cursos complementarios obligatorios a escoger pertenecían al área temática de historia: Historia de América I y II, Historia de México, Historia de China, Historia del Sudeste Asiático, Historia de España, Historia de Japón, así como cualquier otra historia que se impartiera a futuro. 
Tabla 5. Cantidad de cursos por área temática según plan de estudios de $\mathbf{1 9 7 0}$

\begin{tabular}{|l|l|}
\multicolumn{2}{|c|}{ Plan de Estudios 1970} \\
\hline \multicolumn{1}{|c|}{ Área temática } & \multicolumn{1}{c|}{ Cantidad de cursos por área temática en Bachillerato } \\
\hline Artes & 12 \\
\hline Historia & 5 \\
\hline Historia del Arte & 4 \\
\hline Idioma (lengua) & $\begin{array}{l}\text { No se especifica cantidad de cursos, solo cantidad de créditos con los que } \\
\text { se debe cumplir en el tercer (6 lenguas modernas o 5 lenguas clásicas) y } \\
\text { cuarto año (6 en modernas u 8 en clásicas). }\end{array}$ \\
\hline Complementarios & El programa señala que se deben cumplir 10 créditos de estos cursos. \\
\hline Antropología & 2 \\
\hline Letras & 1 \\
\hline Otros & 8 \\
\hline
\end{tabular}

Fuente. Elaboración propia a partir de la información tomada del Consejo Universitario (1970).

Según la información que se presenta, no parece haber una planificación estratégica en aras de establecer una formación académica en Historia del Arte concreta. Más bien, parece ser que este primer plan de estudios fue pensado como una extensión formativa de los cursos del área de Artes Plásticas.

El plan de estudios de 1985 presenta características que distan de la estructura observada en el plan de 1970. Especialmente, en la cantidad y distribución de cursos por área temática. Se da un cambio en el enfoque formativo, ya que sobresale la implementación de más cursos de historia del arte en bachillerato y licenciatura. Los cursos de artes se substituyen por cursos de diseño y cursos introductorios de pintura, escultura, cerámica y grabado (Centro de Evaluación Académica, 1985). Los cursos de historia mantienen un espacio importante dentro del enfoque formativo. La distribución por área temática se presenta en la tabla 6, a continuación: 
Tabla 6. Cantidad de cursos por área temática según plan de estudios de 1985

\begin{tabular}{|l|c|c|}
\hline \multirow{2}{*}{ Área temática } & Cantidad de cursos por área temática según grado académico \\
\cline { 2 - 3 } & Bachillerato & Licenciatura \\
\hline Historia del Arte & 15 & 5 \\
\hline Artes & 9 & 0 \\
\hline Historia & 7 & 2 \\
\hline Idioma & 4 & 0 \\
\hline Antropología & 3 & 1 \\
\hline Letras & 2 & 0 \\
\hline Geografía & 1 & 0 \\
\hline Otros & 7 & 0 \\
\hline
\end{tabular}

Fuente: Elaboración propia a partir de la información tomada del Centro Evaluación Académica (1985).

El plan presenta otro tipo de cursos llamados Lecturas dirigidas. Estos cursos funcionaron como materias de contenido libre. El Sujeto 1 menciona que, estos cursos funcionaban como comodines vacíos que podía utilizar el estudiante para encontrar un espacio de especialización o para vincular lo aprendido con el eventual trabajo final de graduación (Sujeto 1, comunicación personal, 1 de abril del 2017). Para el caso de los cursos destinados a un periodo específico de la historia del arte europea, se necesitaba, únicamente, haber aprobado previamente Introducción a la Historia del Arte impartido en el primer ciclo del primer año de carrera. Al no existir requisitos concretos en esta área, las materias podían ser llevadas en un orden aleatorio, lo que, claramente, pudo afectar el proceso formativo, al generar vacíos y la imposibilidad de establecer relaciones concretas entre elementos clave de la historia del arte y demás disciplinas (Entrevista Sujeto 4, comunicación personal, 19 de mayo del 2017). A pesar de que se percibe la construcción de una finalidad formativa un poco más clara, se mantienen, todavía, vacíos a nivel de contenido curricular y problemas de coincidencias de asignaturas que formen un vínculo educativo entre ellas. Esto provoca confusión al analizar cuál fue la capacidad formativa real del momento, frente a otros campos disciplinarios.

Los planes de estudios del 2007 y del 2013 se presentan como un caso peculiar, debido a que, este último se propuso como una modificación parcial de la estructura de 
cursos del primero. En él se observa una intervención mínima, ya que se sustituyeron únicamente cuatro cursos del área de artes y se introdujeron cursos relacionados con contenidos propios de la historia del arte. Se amplía el espectro de cursos sobre la historia del arte europea, así como cursos de filosofía. Se crean los cursos de Práctica profesional, promoviendo, así, la adquisición de experiencias laborales fuera del ámbito de la academia. La tabla 7 esclarece la cantidad de cursos por área temática que se dieron para el plan del 2007 y los siguientes cambios parciales realizados en el plan del 2013:

\section{Tabla 7. Cantidad de cursos por área temática: plan de estudios del 2007 y 2013}

\begin{tabular}{|l|c|c|c|c|}
\hline \multirow{2}{*}{$\begin{array}{c}\text { Distribución por área } \\
\text { temática }\end{array}$} & \multicolumn{3}{|c|}{ Plan de Estudios 2007 } & \multicolumn{2}{c|}{$\begin{array}{c}\text { Plan de Estudios 2013 } \\
\text { (cambio parcial) }\end{array}$} \\
\cline { 2 - 5 } & Cantidad de cursos por área temática según grado académico \\
\cline { 2 - 5 } & Bachillerato & Licenciatura & Bachillerato & Licenciatura \\
\hline Historia del Arte & 21 & 6 & 24 & 6 \\
\hline Artes & 8 & 0 & 2 & 0 \\
\hline Filosofía & 7 & 1 & 7 & 1 \\
\hline Historia & 5 & 1 & 5 & 1 \\
\hline Antropología & 1 & 0 & 1 & 0 \\
\hline Idioma & 1 & 0 & 1 & 0 \\
\hline Práctica Profesional & 0 & 2 & 0 & 2 \\
\hline Otros & 7 & 0 & 7 & 0 \\
\hline
\end{tabular}

Fuente. Elaboración propia a partir de la información tomada de la Vicerrectoría de Docencia (2007) y del Centro de Evaluación Académica (2013).

Según señala Sánchez (2016), con el cambio curricular del 2007 se dieron modificaciones centradas en la formación educativa. Esto debido a que los nuevos objetivos curriculares buscaron producir "profesionales con amplias capacidades para la investigación y el desarrollo de la teoría y la crítica del arte" (p. 33). Sin embargo, si observamos la estructura de materias propuestas en el plan de estudios, es evidente que no existen cursos dirigidos a la enseñanza y aprendizaje de métodos de investigación, que permitan profundizar en los enfoques y paradigmas que han determinado la producción académica y científica. Existen, únicamente, dos cursos sobre teoría del arte y un curso sobre crítica de arte, por lo que no podemos afirmar que, mientras duró este plan, se dio una amplia formación de profesionales en estos ámbitos, ni en investigación. 
En el nuevo plan de estudios, formalizado a partir del 14 de febrero del 2017 (Centro de Evaluación Académica, 2017), encontramos ya una estructura curricular adecuadamente ordenada, que establece, de forma más concreta, los intereses educativos y al parecer de forma implícita, el eje formativo de la carrera. Este plan contempla una mayor cantidad de modificaciones, tanto a nivel de bachillerato como de licenciatura, lo que da continuidad al proceso de cambio que se venía gestando desde la malla curricular del 2007. Esta transformación deja entrever un interés real por establecer una formación general más integral en su estructura y contenidos, consecuente con los lineamientos de un estudio de la historia del arte académica y tradicional europea. Se integran, además, cursos en la modalidad de optativos, lo que implicó una apertura a nuevos espacios y temáticas de estudio, que van más allá de la enseñanza tradicional del arte europeo. Se proyectó de esta forma, un currículo que posiciona el acto pedagógico hacia perspectivas cercanas al arte oriental, la producción gráfica, la cultura de masas, teorías contemporáneas, entre otras. La tabla 8 presenta los cambios realizados en este plan:

\section{Tabla 8. Cantidad de cursos por área temática según plan de estudios de 2017}

\begin{tabular}{|l|c|c|}
\hline \multirow{2}{*}{\multicolumn{1}{c|}{}} & \multicolumn{2}{c|}{ Plan de Estudios 2017} \\
\hline \multirow{2}{*}{ Área temática } & \multicolumn{2}{c|}{$\begin{array}{c}\text { Cantidad de cursos por área temática según } \\
\text { grado académico }\end{array}$} \\
\cline { 2 - 3 } & Bachillerato & \multicolumn{2}{c|}{ Licenciatura } \\
\hline Historia del Arte & 25 & 9 \\
\hline Historia & 6 & 1 \\
\hline Filosofía & 6 & 0 \\
\hline Artes & 1 & 0 \\
\hline Antropología & 1 & 0 \\
\hline Idioma & 1 & 0 \\
\hline Práctica profesional & 0 & 1 \\
\hline
\end{tabular}

Fuente: Elaboración propia a partir de la información tomada del Centro de Evaluación Académica (2017).

De esta forma, podemos asegurar que el currículo de la carrera de Historia del Arte, actualmente, está constituido por tres ejes. El primero de ellos es el énfasis en la historia del arte occidental. El segundo, un área de filosofía y el tercero un acento en la historia cultural. Los ejes aparecen ordenados en este plan en un sistema de ciclos lectivos pensado de forma cronológica desde el arte, la historia y el pensamiento. 


\section{Categoría 2. Metodología pedagógica}

\section{Subcategoría 2.1 Metodología o estrategia de enseñanza}

Dentro de la carrera de Historia del Arte los docentes han compartido casi que, por igual, el mismo esquema de enseñanza al impartir lecciones. Este se conoce como la clase magistral. Como veremos, ha existido una repetición constante de este método por parte de los docentes. Algunas de las evidencias expuestas en las entrevistas se pueden observar a continuación:

Sujeto 1: No había mucha variedad. El esquema eran clases magistrales. Llegar a escuchar al profesor lo que durara la clase y en mi tiempo era con diapositivas. No había más que eso (comunicación personal, 1 de abril del 2017).

Sujeto 5: Las clases son predominantemente magistrales... ese tipo de enseñanza es vertical y jerárquica. Es magistral (comunicación personal, 17 de junio del 2017).

Cabe destacar que, la clase magistral es un ejercicio metodológico proveniente directo de la tradición pedagógica que permea los modelos de enseñanza dentro de los espacios educativos actuales. Se origina de la enseñanza escolástica de la época Medieval, donde la educación buscaba un control sobre el carácter del individuo. La disciplina era el medio idóneo para educar y la memoria era la base de un currículo centrado en un método vertical, donde el profesor es el emisor y el alumno el receptor del conocimiento (Pinto \& Castro, 2008). Una forma en la cual se estructuraba la información que el profesor tenía que impartir era a través de un medio que el Sujeto 2 y Sujeto 4 llaman "guiones". Al parecer, este mecanismo era básicamente, como lo dice su nombre) un guion estructurado de la clase, donde se utilizaba la información contenida en él para enfatizar las imágenes a partir de las épocas y teorías. Se basaba en este ideal de profesor emisor y alumno receptor del conocimiento.

Sin embargo, el problema de implementar este único método es que el docente no busca fomentar en el estudiante competencias que desarrollen capacidades analíticas e interpretativas sobre los temas y contenidos que se le enseñan. Debido a que, lo que el docente ofrece es un producto dado. Bajo estos criterios, Ortiz (2013) explica cómo esto genera que el individuo sea, simplemente, un elemento pasivo de su propia educación; reproductor del conocimiento otorgado por el docente, e incapaz de ser partícipe de un proceso educativo que busque un aprendizaje significativo en él. Se busca que el estudiante desarrolle más su capacidad memorística y no de análisis, lo que implica una enseñanza centrada en los contenidos, no en el estudiante y sus procesos de aprendizaje. Parece ser que lo que ha caracterizado a la implementación de esta estrategia de enseñanza ha sido el 
apego del docente a la repetición de los mismos lineamientos pedagógicos con los cuales fue educado, ya sea por desconocimiento o simple comodidad. No parece haber una reflexión sobre su propia práctica educativa.

\section{Subcategoría 2.2 Instrumentos de enseñanza}

A partir del uso de la clase magistral, las posibilidades de innovación con respecto a los instrumentos de enseñanza pueden parecer escasas. Es por esto que resulta necesario implementar estrategias didácticas que permitan complementar este método. El uso de diapositivas, presentaciones PowerPoint, así como los documentales y películas, en menor medida, son las herramientas que comparten los docentes de la carrera en su práctica educativa. Todos ellos parten de la necesidad de estudiar los contenidos teóricos, históricos y artísticos representados por la imagen (obra de arte).

La utilización de diapositivas y del archivo de imágenes fue recurrente durante las décadas antes de la aparición del ordenador y de los medios de reproducción digital. Las diapositivas era una especie de pequeñas filminas donde la imagen era impresa de forma individual y proyectada en un espacio determinado. El sujeto 2 resalta el uso de este medio y la creación de un archivo a partir de estos elementos como "proceso investigativo", el cual culmina con la creación de los guiones de clase. Durante su labor como docente, mantuvo la utilización de estos instrumentos (comunicación personal, 20 de abril del 2017).

Por otro lado, se observa la utilización de las presentaciones PowerPoint como el elemento digital que sustituyó a las diapositivas en el esquema magistral. Aquí, se utiliza también la asimilación de lecturas que el profesor asigna al estudiante, para que este complemente lo estudiado en clase. En menor medida, se toma la proyección de documentales o películas como medios que complementan o ensalzan aspectos clave de los contenidos que el docente desarrolla en sus cursos. La utilización de estos medios depende de los intereses pedagógicos de cada profesor, como fue señalado por los sujetos entrevistados.

Todos los instrumentos de enseñanza analizados, anteriormente, ejemplifican el dominio absoluto del esquema de la clase magistral dentro del acto pedagógico llevado a cabo en la carrera de Historia del Arte. De esta forma, se establece un método de "aprendizaje de corte academicista, verbalista, que dicta sus clases bajo un régimen de disciplina a unos estudiantes que son básicamente receptores" (Pinto \& Castro, 2008, p. 3). Así, se afirma la exigencia de un aprendizaje a partir de la memorización de la información que expone el profesor en clase. Es una realidad educativa estática, que se ve permeada, muchas veces, por experiencias que terminan siendo ajenas en su totalidad al alumno. 


\section{Categoría 3. Evaluación de la enseñanza}

\section{Subcategoría 3.1 Instrumentos de evaluación}

La aplicación de estrategias de enseñanza, utilizadas por los docentes para evaluar los contenidos dentro de los cursos de la carrera de Historia del Arte de la UCR parten, indiscutiblemente, de la experiencia o praxis pedagógica dentro del aula. Estas deberían responder a procedimientos dirigidos a alcanzar metas de aprendizaje, a partir de la propuesta de actividades o técnicas que lleven a una evaluación formativa. La experiencia evaluativa dentro de la carrera de Historiad el Arte de la UCR ha respondido a la implementación de un método tradicional educativo que busca: primero medir los conocimientos adquiridos por los estudiantes para, después, evaluar el aprendizaje "real" de estos. Bajo estos criterios, encontramos en la información analizada que, desde una perspectiva histórica, las prácticas evaluativas han mantenido la utilización de un sistema más o menos regular, repetido continuamente por los docentes a lo largo del tiempo. Este sistema de evaluación se basa en tres instrumentos claros: los exámenes de desarrollo, exámenes de diapositivas y la implementación de un trabajo final que concrete los contenidos estudiados. De forma ocasional, dependiendo del criterio del docente, se implementa también los reportes de lecturas y la exposición grupal o individual.

Los exámenes de desarrollo son instrumentos que ponen en práctica una medición de la capacidad retentiva y memorística sobre los contenidos de índole artístico, histórico, social, cultural, entre otros, que se enseñan en las asignaturas de los planes de estudio. Junto a esto, la carrera de Historia del Arte implementa otro tipo de examen de corte memorístico para evaluar el estudio y aprendizaje de las obras de arte, el artista, las técnicas empleadas y el periodo en el cual fueron ejecutada, este se conoce como examen de diapositivas. La utilización de estos dos instrumentos fue mencionada, en repetidas ocasiones, por los sujetos entrevistados, lo cual nos llevó a afirmar lo anterior. Se debe tomar en cuenta que, cada uno de los sujetos entrevistados representa un ejemplo claro de la experiencia educativa de los planes de estudio puestos en práctica.

La ejecución de un trabajo final de investigación busca valorar la comprensión del tema estudiado, la producción de pensamiento crítico y la generación de conocimiento a partir de la investigación académica. Es una herramienta tanto evaluativa como de enseñanza, ya que enfrenta al estudiante con los textos, para generar una nueva producción teórica. Esto solo puede lograrse a través de una formación que busque enseñar al individuo los aspectos metodológicos e interpretativos de investigar, situación que, al parecer, ha representado un vacío que no ha podido solventarse desde la puesta en práctica del primer plan de estudios, hasta el que se encuentra actualmente. Al no existir cursos de metodologías de investigación y de alcances teóricos concretos, la formación resulta incompleta y claramente 
deficiente, más cuando se le exige al estudiante aplicar competencias de las cuales carece.El Sujeto 1 hace un señalamiento bastante interesante sobre el tipo de trabajo de investigación que se solicitaba durante sus estudios de bachillerato. Menciona que, la expectativa de este era simplemente realizar una suerte de monografías o resúmenes con lo más importante y general de la información. Por lo que, carecía de cualquier intento por establecer una estructura investigativa que contuviese introducción, objetivos, aproximación teórica, entre otros (comunicación personal, 1 de abril del 2017).

Las evidencias demuestran cómo se ha mantenido este concepto de la enseñanza tradicional de la historia del arte, donde el academicismo característico de la modernidad, define el paradigma positivista europeo, en el cual se sustenta actualmente esta carrera. Debemos cuestionar, críticamente, la ausencia de otras herramientas o mecanismos evaluativos que vayan más allá de la medición del conocimiento por contenidos. Hay un apego de los docentes, producto de la comodidad y el estatismo, que impide que estos propongan nuevas herramientas evaluativas. También, existe un desconocimiento sobre prácticas pedagógicas y teorías educativas, lo que impide una actualización en el ámbito de la educación. La evaluación debe provocar la generación de competencias claras, que permitan al estudiante lograr utilizar los insumos teóricos, metodológicos o prácticos aprendidos. De esta forma, tiene la posibilidad de desempeñarse fuera del ámbito académico. El docente debe potenciar la creación de conocimiento y, en menor medida, la memorización de este. Buscar una enseñanza que proponga una evaluación por procesos permitiría la práctica de un aprendizaje significativo en aras de una educación superior más integral.

\section{Resultados finales del análisis del modelo pedagógico de la carrera}

Para esclarecer el objeto de estudio de esta investigación, se les hizo a los sujetos entrevistados la siguiente consulta: desde su experiencia dentro de la carrera de Historia del Arte, ¿considera usted que esta posee un modelo pedagógico determinado? A continuación, se presenta en la tabla 9 las respuestas brindadas: 
Tabla 9. Aportes de los sujetos entrevistados sobre el modelo pedagógico

\begin{tabular}{|c|c|}
\hline \multicolumn{2}{|c|}{ ¿Existe un modelo pedagógico en la carrera de Historia del Arte de la UCR? } \\
\hline $\begin{array}{l}\text { Sujeto } 1 \\
\text { No. Primero que nada, históricamente, todos los } \\
\text { que han pasado por las escuelas de artes no } \\
\text { sabemos de pedagogía, partamos de ahí. Creo } \\
\text { que no existía esta reflexión sobre la enseñanza. } \\
\text { (Comunicación personal, } 1 \text { de abril del 2017). }\end{array}$ & $\begin{array}{l}\text { Sujeto } 2 \\
\text { Mirá, no tanto, por una razón. No hay un plan- } \\
\text { teamiento claro y esto se refleja en la carrera de } \\
\text { Historia del Arte. (Comunicación personal, } 20 \text { de } \\
\text { abril del 2017). }\end{array}$ \\
\hline $\begin{array}{l}\text { Sujeto } 3 \\
\text { Como modelo específico no podría ubicarlo. } \\
\text { (Comunicación personal, } 12 \text { de mayo del 2017). }\end{array}$ & $\begin{array}{l}\text { Sujeto } 4 \\
\text { Bueno, vamos ver, así como un modelo peda- } \\
\text { gógico es demasiado ambicioso para que exis- } \\
\text { ta. Realmente no existe. (Comunicación perso- } \\
\text { nal, } 19 \text { de mayo del 2017). }\end{array}$ \\
\hline $\begin{array}{l}\text { Sujeto } 5 \\
\text { No de una manera consciente. (Comunicación } \\
\text { personal, } 17 \text { de junio del 2017). }\end{array}$ & $\begin{array}{l}\text { Sujeto } 6 \\
\text { No, no creo que la carrera tenga un modelo } \\
\text { pedagógico determinado para la enseñanza. } \\
\text { (Comunicación personal, } 14 \text { de noviembre del } \\
\text { 2017). }\end{array}$ \\
\hline
\end{tabular}

Fuente. Elaboración propia a partir de la información suministrada en las entrevistas.

A partir de la información presentada, nos damos cuenta de varias situaciones. La primera de ellas es muy clara, ninguno de los sujetos entrevistados consideró que la carrera de Historia del Arte de la UCR, en sus distintos planes, fuese estructurada bajo un modelo pedagógico. Esto, inmediatamente, implica que la praxis educativa no responde a una estructura organizada que parta de una reflexión en conjunto con los actores responsables de ejecutarla (docentes pertenecientes a la cátedra, Unidad Académica, entre otros). Más bien, se trata de una práctica de carácter individual de la enseñanza que, al parecer, no ha permitido que se dé una cohesión a lo interno de la elaboración y ejecución de los programas.

Se comprende que, se ha partido, desde la creación de la carrera, de una enseñanza basada en la perspectiva de la academia tradicional del arte europeo, observada en el paradigma del positivismo, que ha ido creciendo con cada modificación curricular. El problema es que, a nivel formal, no pudo identificarse ningún insumo que brindase premisas claras del porqué y para qué se está formando al estudiante de Historia del Arte. No se evidencia una problematización de las estructuras tradicionales de aprendizaje, ni una meta profesional ordenada, establecida y reflexionada, lo cual afecta, directamente, la definición del perfil profesional del próximo historiador del arte. 
Otra condición que ha determinado la ausencia de un modelo pedagógico dentro de la carrera, parte del trabajo independiente realizado por cada docente a lo interno. Cuando nos referimos al trabajo en conjunto de los docentes de la cátedra, parece haber una especie de divorcio entre ellos, lo que ha impedido que se realicen esfuerzos concretos por establecer una meta o finalidad formativa clara a través de los años. Esto podría reflejar una falta de evaluación autoconsciente de su proceso educativo. No hay una perspectiva que englobe el acto pedagógico y que demuestre cuál es el objetivo de la formación en Historia del Arte en la UCR.

El problema no es que la práctica docente parta de un esquema particular desarrollado por cada profesor. Lo que sucede es que este no procede de un ordenamiento de las herramientas de planeamiento y sistematización del proceso educativo. Debido a esto, la enseñanza en el aula se convierte en una repetición del método de la clase magistral y su instrumentalización a partir de los ejercicios evaluativos tradicionales. Al no existir este proceso de reflexión y de autoevaluación se observa, entonces, una serie de componentes del acto pedagógico representados por las categorías y subcategorías. Estas se ponen en práctica por una tradición de enseñanza: "porque así fue como yo lo aprendí, así funciona y hasta que nadie me diga que hay otra mejor, porque pensar en otra mejor" (Entrevista Sujeto 1, comunicación personal, 1de abril del 2017). Por esto, podemos afirmar que no puede haber un modelo pedagógico establecido, que parta de criterios de enseñanza sólidos y de un paradigma educativo, si este no ha sido formulado, expuesto, analizado y reflexionado en conjunto.

Por un lado, se identificaron ciertas similitudes entre el acto o práctica pedagógica de los docentes de la carrera de Historia del Arte y elementos teórico-prácticos de los modelos pedagógicos de enseñanza tradicional y conductista. Este modelo proveniente de la escolástica de la época Medieval, parte de un modelo basado en la enseñanza de contenidos, donde la formación del estudiante es disciplinar. La relación profesor-alumno es meramente vertical, poco reflexiva y basada en una jerarquía del conocimiento (poder). La forma en la cual se transfiere el conocimiento parte de la transmisión de los contenidos, a partir de la clase magistral, donde el estudiante recibe los insumos ya establecidos; el aprendizaje se da con base en la memorización, la repetición, y la ejercitación (Pinto \& Castro, 2007, p. 4). Hay una construcción muy cercana de los elementos estudiados que constituyen el acto pedagógico dentro de la carrera de Historia del Arte y los insumos teóricos y metodológicos que componen este modelo tradicionalista. Por otro lado, encontramos el llamado modelo conductista, que comparte, también, parte de la herencia del modelo tradicionalista. Surge en el siglo XIX como una teoría de aprendizaje que se desarrolló paralela al auge del capitalismo, con su creciente racionalización y planeación económica. Se establece bajo una construcción donde se moldea meticulosamente la conducta productiva del individuo (Pinto \& Castro, 2008, p. 4). 
Este esquema de aprendizaje parte de un proceso educativo que busca moldear las conductas de los individuos, de forma adecuada y técnica, para así cumplir con una estandarización absoluta de los componentes educativos. Para el caso de este estudio, la estandarización no se comparte, ya que para que se dé, debe existir un control absoluto del acto pedagógico, lo que involucraría una formulación de procedimientos ordenados y reflexionados, aspecto que no existe dentro de la carrera de Historia del Arte. Las similitudes que podemos encontrar entre la práctica educativa docente de la carrera y este modelo son las siguientes: el profesor es el elemento activo de la dinámica dentro del aula. Es el trasmisor de conocimientos, autoritario, rígido, controlador, no espontáneo; se encarga de ejecutar procesos ya preestablecidos. A la par de esto encontramos al estudiante, elemento pasivo y reproductor de conocimientos.

\section{Conclusiones}

Esta investigación buscó evidenciar cómo se han comportado los procesos curriculares formativos que constituyeron lo que, inicialmente, comprendimos como el modelo pedagógico de la Carrera de Historia del Arte de la Universidad de Costa Rica, desde 1970 hasta el 2017. Como hemos constatado, en cuarenta y siete años de existencia, la carrera se ha ido construyendo sin una idea clara sobre cómo deben desarrollarse los procesos curriculares, de forma que estos puedan responder, directamente, a los intereses pedagógicos y al perfil profesional que ofrece la carrera. La revisión documental y las evidencias tomadas de las entrevistas constataron que, estos procesos nunca fueron articulados como un sistema ordenado y reflexionado desde la práctica administrativa y educativa de la carrera. Por lo tanto, queda claro que no existe, ni ha existido realmente un modelo pedagógico definido. Ninguno de los planes de estudio llegó a contemplar, en su estructura curricular, cursos relacionados con metodologías de investigación y alcances teóricos aplicados. Esto implica que el estudiante carece de las competencias necesarias para realizar procesos investigativos exitosos y coherentes con las necesidades específicas de una disciplina como la historia del arte.

La estructura metodológica y evaluativa reproducida responde, principalmente, a una práctica personal e individual del docente y no a un consenso establecido que formule pautas concretas, para la estructuración modular de la carrera. Sobre estos apartados encontramos que existe una continua reproducción de lineamientos típicos de la educación tradicional, donde el esquema de la clase magistral repite un patrón lineal, que no ha permitido el cambio. Su práctica educativa refleja una subordinación a la réplica de los contenidos de los programas de los cursos, lo que ha generado una carencia de autorreflexión y evaluación consiente del propio proceso educativo. Esto, aunado a una estructura curricular que apenas hacia el 2017 se logró articular cronológicamente y con una finalidad más 
clara, ha provocado que el quehacer académico, pedagógico y docente se trabajen de forma desarticulada.

La estructura curricular o pedagógica que ha compuesto los distintos planes de estudio de la carrera comparten ciertos elementos y herramientas de los modelos tradicional y conductista. Sin embargo, no existen evidencias suficientemente claras para afirmar que se hayan propuesto y ejecutado continuamente de forma intencionada. Se parte de una tradición de la educación formal, donde el profesor utiliza las mismas herramientas formativas con las cuales fue educado. Se trata, básicamente, de la repetición complaciente de un método de enseñanza y de construcción curricular. La práctica docente es reflejo de una actividad individual e inconsciente, poco reflexionada, donde se demuestra un desconocimiento absoluto sobre procesos pedagógicos claros. Esto parece suceder debido a una relación causal entre los contenidos tradicionales de la historia del arte y lo que podríamos llamar una herencia del método educativo, que pasa de profesor a alumno, sin revisión ni posibilidad de cambio y, así, sucesivamente.

\section{Referencias}

Centro de Evaluación Académica. (1985). Malla Curricular de 1985 para la Carrera Bachillerato y Licenciatura en Historia del Arte. San José, Costa Rica: Universidad de Costa Rica.

Centro de Evaluación Académica. (2013). Malla Curricular del 2013 para la Carrera Bachillerato y Licenciatura en Historia del Arte. San José, Costa Rica: Universidad de Costa Rica.

Centro de Evaluación Académica. (2017). Malla Curricular del 2017 para la Carrera Bachillerato y Licenciatura en Historia del Arte. San José, Costa Rica: Universidad de Costa Rica.

Consejo Universitario. (28 de setiembre de 1970). Acta de la Sesión No 1790. Aprobación del primer plan de estudios para la carrera de Historia del Arte. San José, Costa Rica: Universidad de Costa Rica.

Consejo Universitario. (31 de mayo de 1972). Acta de la Sesión No 1832. Aprobación de inclusión de nuevos cursos de repertorio para la carrera de Historia del Arte. San José, Costa Rica: Universidad de Costa Rica.

Efland, A. (2002). Una historia de la educación del arte. Tendencias intelectuales y sociales en la enseñanza de las artes visuales (trad. R. Vilá Vernis). España: Paidós.

Gómez Hurtado, M. \& Polania, N. (2008). Estilos de enseñanza y modelos pedagógicos: un estudio con profesores del Programa de Ingeniería Financiera de la Universidad Piloto de 
Colombia [Tesis para optar por el grado de Maestría en Docencia]. Bogotá, Colombia, Universidad de La Salle. Recuperado de http://repository.lasalle.edu.co/bitstream/handle/10185/1667/T85.08\%20G586e.pdf

Hernández, R., Fernández, C. \& Baptista, P. (2010). Metodología de la Investigación. México: Mc Graw-Hill.

Mora, A.I. (2004). La evaluación educativa: conceptos, períodos y modelos. Actualidades Investigativas en Educación, 4(2), 1-28. Recuperado de https://revistas.ucr. ac.cr/index.php/aie/article/view/9084/17481

Ortiz, A. (2013). Modelos pedagógicos y teoría del aprendizaje. Colombia: Ediciones la U. Recuperado de https://es.scribd.com/document/246403512/07-LIBRO-Modelos-pedagogicos-y-Teorias-Del-Aprendizaje-Alexander-Ortiz

Pinto, A. \& Castro, L. (2008). Los modelos pedagógicos. Revista Ideales, 7, 1-10. Recuperado de https://pedroboza.files.wordpress.com/2008/10/2-2-los-modelos-pedagogicos.pdf

Sánchez-Zumbado, J. (2016). Arte y Curriculum: la Escuela de Artes Plásticas de la Universidad de Costa Rica (1897-2015). Revista Humanidades, 6(1), 1-40. Recuperado de http://revistas.ucr.ac.cr/index.php/humanidades/article/view/24963/25158

Vicerrectoría de Docencia (2007). Justificación curricular del Plan de estudios del 2007. San José, Costa Rica: Universidad de Costa Rica.

Vicerrectoría de Docencia (2007) Resolución VD-R-8126-07. Malla Curricular 2007 Carrera Bachillerato y Licenciatura en Historia del Arte. San José, Costa Rica: Universidad de Costa Rica.

Vicerrectoría de Docencia (2013). Resolución VD-R-8909-2013. Cambio parcial a la malla curricular y creación de nuevos cursos para la Carrera Bachillerato y Licenciatura en Historia del Arte. San José, Costa Rica: Universidad de Costa Rica. 